\title{
Time Synchronization for Mobile Wireless Sensor Network
}

\author{
Asma Khan, Aftab Ahmed Memon, and Abdul Aziz Memon
}

\begin{abstract}
Existing time synchronization protocols for Wireless Sensor Networks (WSN) have already focused on lot of time synchronization issues like accuracy, energy efficiency, security and time synchronization itself etc for static wireless sensor networks but best of our knowledge time synchronization for mobile wireless sensor networks has not been fully explored yet, so there is need to design protocols that can assist to time synchronize mobile wireless sensor networks in a simple way to achieve better time synchronization accuracy.

In this paper we propose mathematical model that is basically an extension of pair-wise time synchronization protocol and it aims to synchronize mobile wireless sensor node with the help of nearby static wireless sensor node. This model can be used to achieve network wide time synchronization. Proposed model has kept the track of direction and velocity of mobile nodes. Proposed protocol has been simulated and results are compared with existing pair-wise time synchronization protocol. Results show that pair-wise time synchronization protocol introduces significant error that will keep increasing with the moving velocity of mobile node. In contrast proposed algorithm performed very well in terms of accuracy, energy and computational cost and was able to synchronize mobile nodes with the help of static sensor nodes successfully.
\end{abstract}

Index Terms-Mobile sensors, time synchronization, wireless sensor network.

\section{INTRODUCTION}

In WSN individual nodes sense and control physical parameters to interact with the environment; these nodes need to collaborate with each other to complete their tasks. Usually, this collaboration between sensor nodes can be enable by using wireless communication. Moreover, if the nodes are not having such a network, at least they hold some of the functionality like computation; sensing or controlling and wireless communication. WSN are powerful in a sense that many of the different real-world applications are supported by sensor nodes. Some of the few popular applications of WSN are event detection, tracking, temperature monitoring, humidity sensing, pressure detection, health care, disaster monitoring etc.

The sensor nodes are usually scattered in a sensor field as shown in Fig. 1. [1]. Sensing task can be done by the sensor nodes and the aggregation of data is done by the sink node.

Manuscript received July 15, 2013; revised September 16, 2013.

Asma Khan is with Department of Computer Science, Isra University Hyderabad. (e-mail: asma.ansari@isra.edu.pk).

Aftab Ahmed Memon is with Department of Telecommunication Engineering, Mehran University of Engineering and Technology, Jamshoro, Pakistan (e-mail: memon_aftab@yahoo.com).

Abdul Aziz Memon is with Department of Electrical Engineering, Sukkur Institute of Business Administration, Pakistan (e-mail: azizsheraz@yahoo.com).
Scattered senor nodes have abilities to gather date and route to sink node via single or multi-hop communication as shown in Fig. 1. [1].

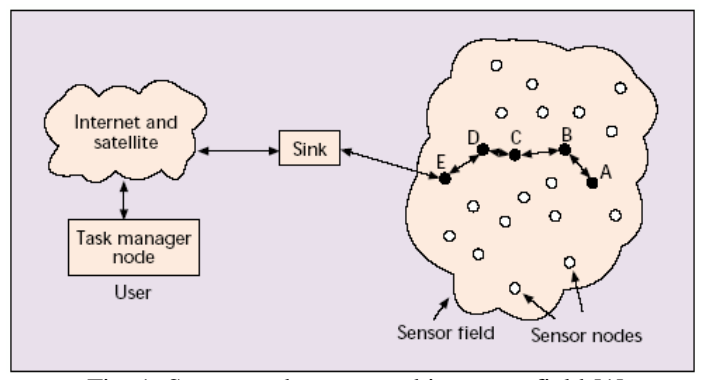

Fig. 1. Sensor nodes scattered in sensor field [1]

Each sensor node is consists various units such as sensing unit, processing, communicating, power unit, mobilizer, location finding unit etc as shown in Fig. 2. [1].

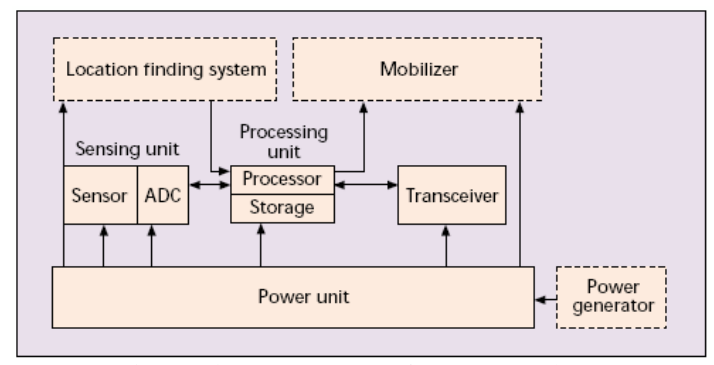

Fig. 2. The components of a sensor node [1].

Due to energy and data handling constraints, lot of research is carried out in the field of WSNs to make them efficient in the sense of routing, scheduling, localization, time synchronization etc. This research paper focuses on the area of time synchronization for mobile WSNs. Our designed model is used to synchronize of mobile nodes with the help of static nodes. This work is basically an extension of pair-wise synchronization algorithm which aimed to synchronize static sensor nodes and after comparing modified algorithm with previous work, we have found that precision in mobile wireless sensor networks cannot be achieved due to constant propagation delay assumption during synchronization process and while not considering the mobility of sensor nodes, which will introduce significant error that will keep on increasing with the increasing speed of mobile node. In contrast modified algorithm performed very well and synchronized mobile sensor nodes efficiently throughout the synchronization process.

\section{TIME SYNCHRONIZATION}

In multi-hop ad-hoc wireless networks like sensor networks 
time synchronization is an important area of concern. However, not only WSN applications but also for many networking protocols used in sensor networks need accurate timings [2]. Sometimes it is essential for a sensor node to know that at what time any particular event has occurred in a controlled field, High accuracy of local clocks is needed to ensure interaction of the network nodes. These sensor nodes coordinate their efforts and work together for achieving complex identification/sensing task. One example of this co-ordination is data merger and fusion in which data should be collected from various active nodes and aggregated, accumulated into useful results [1]. For instance, in vehicle tracking system, location of vehicle being tracked is reported by the sensor nodes. In this case, if the sensor nodes are not synchronized (i.e. they lack a common timescale) their estimations will be inaccurate. and lifetime of networks can be increased if power saving schemes use synchronization techniques, Time division multiple access (TDMA) and other scheduling algorithms divide transmission media into time domain and eliminate collisions and save energy so it has been an increasing research focus on designing synchronization algorithms specifically for sensor networks [2]. Computing devices are mostly outfitted with an oscillator [1] assisted clock. According to [2] every individual sensor in a network has its own clock. Ideally, the clock of a sensor node should be configured such that $C(t)=t$, where $t$ stands for the ideal or reference time. On the other hand, due to faults and inadequacies of the clock oscillator, a clock will go away from the ideal time and have difference in timings even if it is initially perfectly tuned [2] and it can be modeled as given in equation 1.

$$
C_{i}(t)=\theta+f . t
$$

where $\theta$ and $f$ are called clock offset (i.e. phase difference and variation) and clock skew (i.e. frequency difference and variation) respectively. From equation 1, the clock association of two Nodes $A$ and $B$ can be given as in equation 2.

$$
C_{B}(t)=\theta^{A B}+f^{A B} \cdot C_{A}(t)
$$

where $\theta^{A B}$ and $f^{A B}$ are the relative clock difference and frequency difference between nodes $A$ and $B$ and graphical representation is shown in Fig. 3. [2].

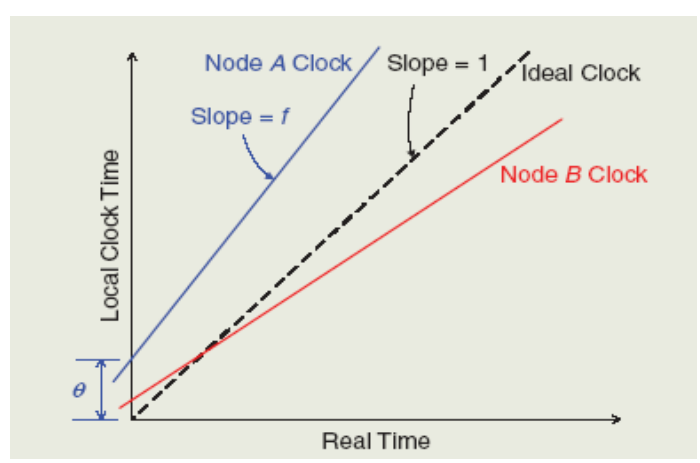

Fig. 3. Clock model of sensor nodes [2]

Seemingly, if two clocks are get synchronized and faultlessly tuned then $\theta^{A B}=0$ and $f^{A B}$. If node $A$ is considered as a reference node then the clock synchronization has to approximate and estimate these two parameters in such a way that Node $B$ adjusts its own clock or translates its timing information to the time scale of Node $A$ whenever needed. The local clock time at any chosen "reference" node can be the global time to which all nodes must be synchronized. Truly the time synchronization problem is a problem of estimation of the skews (drifts) and offsets of every node. Clock parameters are area of research and discussion which are easily affected due to various environmental changes and other external factors/effects such as voltage change, hardware aging, atmospheric pressure and temperature [1].

To achieve Clock synchronization in WSN needs transferring and exchanging a set of timing messages to the target sensors [2]. Time stamp information that is contained in these timing messages is calculated by the transmitting sensors. To solve the problems and issues of time synchronization related wireless sensor networks, various protocols have been proposed [3]-[16]. Most of these protocols aim to time synchronize static wireless sensor networks with accuracy, security, energy efficiency etc, but they have not discussed issue of time synchronization for mobile wireless sensor networks.

\section{TRADITIONAL SYNCHRONIZATION ALGORITHMS}

\section{A. Pairwise Time Synchronization}

Pairwise time synchronization algorithm is extremely simple and computationally efficient and used to synchronize any pair of static sensor nodes, and consider one of the nodes as a reference node among two nodes. Better radio characteristics of sensor node can help to achieve accuracy and according to algorithm only three messages will be exchanged between nodes [4]. In Fig. 4 it is shown that there are two nodes $j$ and $k$ that are supposed to synchronize with each other.

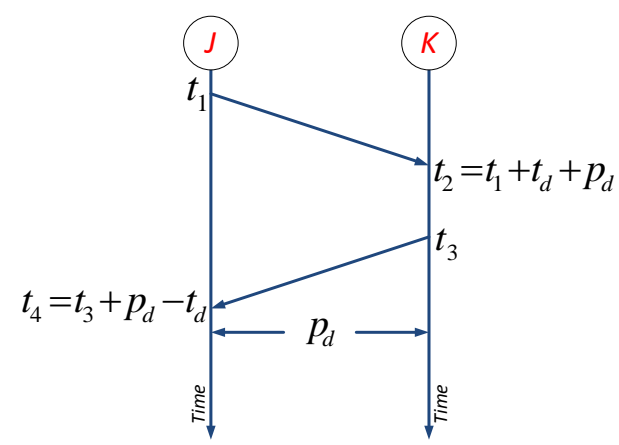

Fig. 4. Synchronization between node $J$ and $K$ using pairwise synchronization technique.

Synchronization of local time of Nodes $J$ and $K$ takes place, when exchange of two packets will be done with the following procedure:

The first packet will be transmitted by Node $J$ with a timestamp $t_{1}$ with respect to its local time.

When Node $K$ receives the first packet, it records its local time that is $t_{2}$ where

$$
t_{2}=t_{1}+t_{d}+p_{d}
$$


$P_{d}$ is the transmission time (propagation delay) taken by packet to propagate from one node to other, which is function of the distance between the nodes and signal propagation characteristic and it is unknown. $t_{d}$ is the offset between two nodes.

Next, a second packet to node $J$ is transmitted by node $K$, which contains $t_{1}$ and $t_{2}$. This packet is also time stamped by node $K$ at time $t_{3}$.

The second packet has been received by Node $J$, where

$$
t_{4}=t_{3}+p_{d}-t_{d}
$$

At node $J$ by subtracting and adding equation 4 with equation 3 will result in offset $t_{d}$ and $P_{d}$ respectively.

$$
\begin{aligned}
& t_{d}=0.5 \times\left(t_{2}-t_{4}-t_{1}+t_{3}\right) \\
& p_{d}=0.5 \times\left(t_{2}+t_{4}-t_{1}-t_{3}\right)
\end{aligned}
$$

Once node $J$ has calculated the offset and propagation delay, the two nodes will get synchronized, however a third message is required if offset need to be communicated to node $K$.

\section{B. Reference Broadcast Time Synchronization}

Reference broadcast synchronization (RBS) is another synchronization scheme used in sensor networks that was proposed by Elson and Estrin [5], they offer receiver to receiver synchronization model. In this technique a reference broadcast does not contain an explicit timestamp; instead, receivers use its arrival time as a point of reference for comparing their clocks.

\section{Timing-Sync Protocol for Sensor Networks}

A network wide time sync protocol was proposed by Ganeriwal et al. for sensor networks which was called Timing-Sync Protocol for Sensor Networks (TPSN) [6]. They divided their work in two steps. Level discovery phase and synchronization phase. During first step of level discovery a hierarchical topology of the entire network is created and a level is assigned to each and every node, and level 0 is assigned to root node. In the second phase, a node of level $\mathrm{k}$ is synchronized to a node of level k-1. The whole network can get synchronized by synchronizing all nodes to the root node.

\section{Lightweight Tree-Based Synchronization}

Lightweight Tree-based Synchronization (LTS) was proposed by Greunen and Rabaey [4] and it is distinguished from other work in the sense that the aim is not to maximize accuracy, but to minimize the complexity of the synchronization. Thus the needed synchronization accuracy is assumed to be given as a constraint, and the target is to devise a synchronization algorithm with minimal complexity to achieve given precision.

\section{Problem Statement ANd Proposed Algorithm}

Most of the algorithms that are already proposed for time synchronization in wireless sensor networks don't consider mobility of sensor nodes.
There are various applications in which wireless sensor nodes are mobile; such as vehicle tracking system, telemedicine where sensor nodes attached with mobile patient; those nodes continuously monitor health conditions. Already presented algorithms that are suitable for static wireless sensor networks are not appropriate to use for the synchronization of mobile wireless sensor networks. Therefore a synchronization technique that can work to synchronize mobile sensor networks is needed.

The main idea behind this research is to design and develop a time synchronization algorithm that can be effectively utilized by a mobile node to synchronize it with the static node.

Designed mathematical model is an extension and modified form of Pair-wise synchronization algorithm which did not consider mobility of sensor nodes. When nodes are mobile, pair wise synchronization algorithm fails to find out exact time difference between two nodes because of varying distances between two nodes.

Proposed algorithm considered the mobility of nodes. In suggested algorithm node $J$ is considered as static node and node $K$ is mobile with some known constant velocity, moving either away or towards node $J$. For that purpose equation 4 of pairwise algorithm is modified to support the mobility of node $K$. In Fig. 5. it is shown that node $\mathrm{k}$ is moving away from node $j$ with constant velocity.

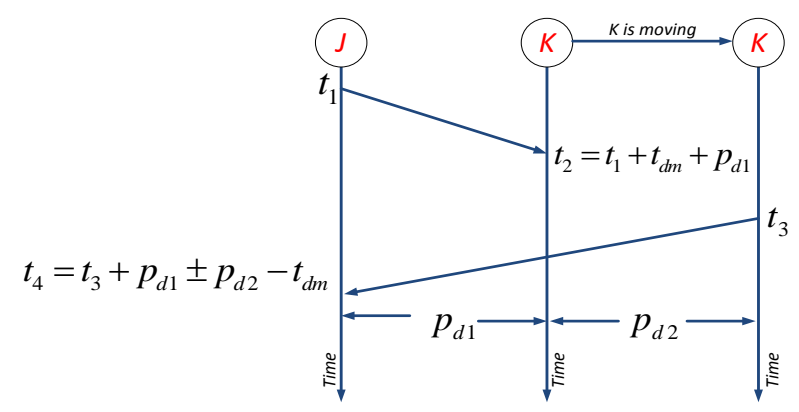

Fig. 5. Pair wise time synchronization with mobile node.

Here $t_{d m}$ is time difference between mobile and static node, $P_{d 1}$ are $P_{d 2}$ two propagation delays. It can be seen that $t_{4}$ is the function of additional propagation time $P_{d 2}$ which is caused due to mobility of sensor node $K . P_{d 2}$ is the function of velocity and direction of mobile node. Modified equation for $t_{4}$ can be given as equation 7 .

$$
t_{4}=t_{3}+p_{d 1} \pm p_{d 2}-t_{d}
$$

$P_{d 2}$ is positive when node $K$ is moving away from node $J$ and $P_{d 2}$ is negative when node $K$ is moving towards node $J$. Value of $P_{d 2}$ can be calculated by using the distance formula as follows.

Distance covered $=$ velocity $\times$ time

$$
\begin{gathered}
s=v \times t \\
s=v_{\text {light }} \times p_{d 2}
\end{gathered}
$$

$P_{d 2}$ is the additional propagation delay caused due to mobility of node, $v_{\text {light }}$ is the seed of light through which signal 
will be transmitted between nodes and $s$ is distance covered during $P_{d 2}$ and that distance can also be given by equation 10 .

$$
s=v_{k}\left(t_{3}-t_{2}\right)
$$

After putting the value of equation 10 in equation 9, we get.

$$
p_{d 2}=v_{k}\left(t_{3}-t_{2}\right) / c
$$

where $v_{k}$ is the velocity of node $K$ and $c$ is the velocity of light. Adding equation 4 and 7 and using equation 11, equation of time offset with mobility between two nodes can be given as.

$$
t_{d m}=0.5 \times\left\{\left(t_{2}-t_{4}-t_{1}+t_{3}\right) \pm v_{k}\left(t_{3}-t_{2}\right) / c\right\}
$$

By implementing this equation mobile node will be able to synchronize itself with the nearby static node with more accuracy than simple pairwise synchronization algorithm.

\section{Simulation SCENARIO AND RESUlts}

Both pairwise and modified pairwise with support of mobile time synchronization are compared in terms of calculation of time difference between two nodes, while one node is static and other is moving away with constant velocity. 1) Will consider static node as a reference node with the initial time difference of $1 \mu \mathrm{sec}$.

2) Node $K$ is moving away from reference node $J$ with constant velocity.

Initially two nodes are considered in the scenario but the same methodology will be used for network wide synchronization. Velocity of mobile node changed from $10 \mathrm{~m} / \mathrm{sec}$ with addition of $10 \mathrm{~m} / \mathrm{sec}$ up to $300 \mathrm{~m} / \mathrm{sec}$. Sensor nodes have enough transmission range to communicate with each other during varying speed.

For simulation results equation 5 and 12 are used to find time difference between two nodes. Where equation 5 will be giving results for time difference between two without support of mobility and equation 12 will be giving results for time difference between two nodes with mobility support.

Implementation results of already present synchronization algorithms for mobile wireless sensor nodes in Fig. 6. show how error is keep growing with increasing speed of mobile node, moreover we can say that pair wise time synchronization algorithm without mobility support can't achieve high accuracy in case if the sensor node is mobile because of the varying propagation delay during synchronization process, which cause significant errors. Synchronization error will keep on increasing not only with increase in the velocity of sensor nodes in particular direction but also with increase in communication hops. Fig. 6. shows results of time difference between two nodes using already present pair wise time synchronization and modified suggested time synchronization algorithm.

Algorithms Calculation of time offset between two nodes in the above figure vertical axis is showing difference of time between reference node and the mobile node that suggested algorithm needs to maintain as $1 \mu$ sec regardless of moving velocity of mobile node. The horizontal axis of resulted graph is showing the distances covered by mobile node while moving away from the reference node.

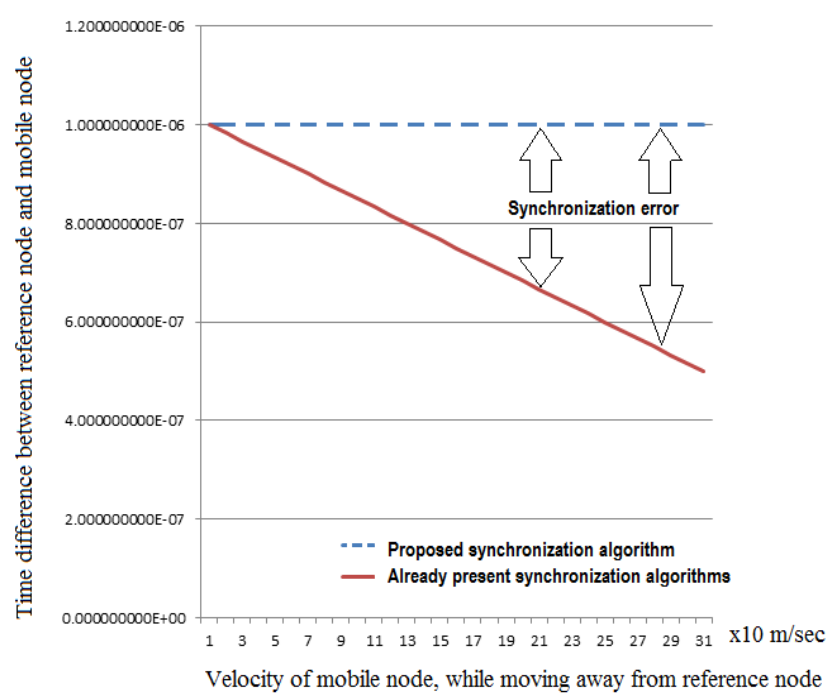

Fig. 6. Synchronization error with proposed and already present.

In Results it is shown that two nodes with actual time difference of $1 \mu \mathrm{sec}$ are synchronized. With proposed algorithm; value of time difference between two nodes is always obtained as $1 \mu \mathrm{sec}$, which is the actual time difference between two nodes. It means synchronization error is zero with suggested modified algorithm and modified algorithm is able to achieve synchronization between mobile sensor nodes successfully and if already present pairwise synchronization algorithm is implemented for synchronization of mobile nodes then synchronization error is increasing with increase in velocity of mobile nodes.

\section{FUTURE WORK}

The future work will be conducted with the consideration of multi-hop mobile network with random deployment of sensor nodes moving in different directions. In this work we have assumed a wireless sensor network in which mobile node was moving away or towards static node and with different speed and direction and we designed a mathematical algorithm for the time synchronization of these nodes. In future we will be designing a mathematical model for the time synchronization of mobile node if mobile node is moving randomly nearby static node with different speed and directions. More analysis parameters will be taken into consideration to be used in simulation model.

Another work that could be conducted in future is to synchronize two sensor nodes if both of these nodes are mobile and moving with not only different speeds but different accelerations and direction, and finally we will be synchronizing whole network with different speeds, accelerations, and directions of nodes.

\section{REFERENCES}

[1] Ian F. Akyildiz, Weilian Su, Yogesh Sankarasubramaniam, and Erdal Cayirci, "A Survey on Sensor Networks," IEEE Communications Magazine, vol. 40, issue 8, pp. 102-114, August 2002. 
[2] W. Yikchung, Q. Chaudhari, and E. Serpedin, "Clock synchronization of wireless sensor network," IEEE Signal Processing Magazine, January 2011.

[3] J. Elson, D. Estrin, "Time synchronization for WSN," in Proc. IEEE 15th International Conference on Parallel and Distributed Processing, 2001.

[4] J. V. Greunen, and J. Rabaey, "Lightweight time synchronization for sensor networks," in Proc. the 2nd ACM International Conference on Wireless Sensor Networks and Applications, 2003.

[5] J. Elson, L. Girod, and D. Estrin, "Fine-Grained time synchronization using reference broadcasts," in Proc. the Fifth Symposium on Operating Systems Design and Implementation, Boston, MA, December 2002

[6] S. Ganeriwal, R. Kumar, and M. Srivastava, "Timing sync protocol for sensor networks," presented at ACM SenSys, Los Angeles, November 2003.

[7] M. L. Sichitiu and C. Veerarittiphan, "Simple, accurate time synchronization for wireless sensor networks," presented at IEEE Wireless Communications and Networking Conference, WCNC 2003.

[8] F. Sivrikaya, and B. Yener, "Time synchronization in sensor networks: A survey," IEEE Network, vol. 18, no. 4, August 2004.

[9] K. Sun, P. Ning, and C. Wang, "Secure and resilient clock synchronization in wireless sensor networks," IEEE Journal on Selected Areas in Communication, vol. 24, no. 2, February 2006.

[10] S. Ganeriwal, R. Kumar, and M. B. Sirvastava, "Secure time synchronization service for sensor networks," in Proc. the 4th ACM Workshop on Wireless Security, Cologne Germany, 2005.

[11] M. Maróti, B. Kusy, G. Simon, and Á. Lédeczi, "Flooding time synchronization," in Proc. the 2nd International Conference on Embedded Networked Sensor Systems, Baltimore, MD, USA, 2004.

[12] Y. S. Liu, J. Li, and M. Guizani, "Lightweight secure global time synchronization for wireless sensor networks," in Proc. IEEE Wireless Communications and Networking Conference, 2012, pp 2312-2317.

[13] F. Y. Ren, C. Lin, and F. Liu, "Self-Correcting time synchronization using reference broadcast in wireless sensor network," IEEE Wireless Communications, vol. 15, pp. 79-85, 2008.

[14] X. L Yin, W. D. Qi, and F. Fu, "ASTS: An agile secure time synchronization protocol for wireless sensor networks," presented at IEEE International Conference on Wireless Communications, Networking and Mobile Computing, 2007.

[15] H. Cho, J. Jung, B. Cho, Y. W. Jin, S. W. Lee, and Y. J. Baek, "Precision time synchronization using IEEE 1588 for wireless sensor networks," presented at IEEE International Conference on Computational Science and Engineering, 2009.

[16] K. L. Noh, E. Serpedin, and K. Qaraqe, "A new approach for time synchronization in wireless sensor networks: Pairwise broadcas synchronization," IEEE Transactions on Wireless Communications, vol. 7, issue 9, pp. 3318-3322, 2008.

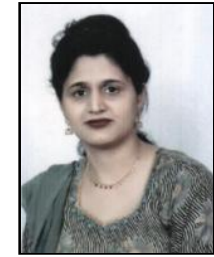

Asma Khan is an assistant professor at Department of Computer Science, Isra University Hyderabad. She obtained her Bachelor of Engineering degree from Mehran University and received Master's degree in information technology from Mehran University, Hyderabad Pakistan. She has authored/co-authored 10 publications. She has done her research activities in the fields including: Mobile AdHoc networks, WSNs, data communication networks and Databases. She has supervised around 10 academic projects in Master's degree programs. She is planning to be a candidate for PHD. She has been external examiner at IBA Sukkur and MUET for various departments. She is registered member of Pakistan Engineering Council.

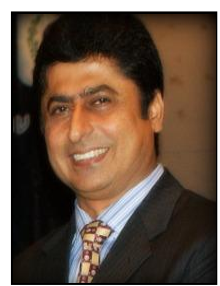

Aftab Ahmed Memon is the professor and chairman at Department of Telecommunication Engineering, Mehran University of Engineering and Technology, Jamshoro. He obtained his doctorate degree from Miyazaki University Japan and Master's degree from Louisiana State University, USA. He has authored/co-authored 38 publications, edited three books. His research interests include: antennas, data communication networks (wired and wireless) and

photonic devices.

Dr. Memon has supervised around 50 academic/industry oriented projects in doctorate and Master's degree programs. Dr. Memon has a strong linkage with the industry and is invited as an expert on many academic and industrial forums.

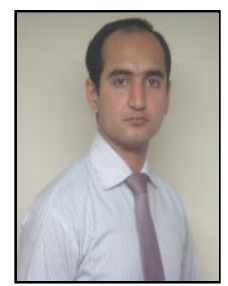

Abdul Aziz Memon received MS degree in Electronic \& Electrical Engineering from Hanyang University South Korea in 2010 and BS degree in Telecommunication Engineering from Mehran University of Engineering and Technology Pakistan in 2007. Currently he is PhD candidate at the department of Electronic and Computer Engineering Hanyang University South Korea as well as he is Assistant Professor at the department of Electrical Engineering Sukkur Institute of Business Administration Pakistan. He has more than 6 years of combined experience in the field of research, teaching and industry. $\mathrm{He}$ is registered member of Pakistan Engineering Council since 2007. He was also awarded scholarships from government of Pakistan to pursue MS and $\mathrm{PhD}$ level education. His research interests mainly focus on wireless sensor networks, wireless ad hoc networks, building automation, smart grid cellular networks etc. 